\title{
ANNOUNCEMENT
}

\section{NEW MEMBERS OF THE \\ PALEONTOLOGICAL SOCIETY}

In accordance with the By-laws of The Paleontological Society, the names of new members are listed below:

ANDERSON, WAYNE, Department of Earth Science, University of Northern Iowa, Cedar Falls, IA 50614.

ANDO, HISAO, Institute of Earth Science, School of Education, Waseda University, Nishiwaseda Shinjuku, Tokyo 160, Japan.

ARCHER, MICHAEL, School of Zoology, University of New South Wales, P.O. Box 1, Kensington NSW 2033, Australia.

BARNOSKY, ANTHONY D., Carnegie Museum of Natural History, Vertebrate Fossils, 440 Forbes Ave., Pittsburgh, PA 15213.

BIRD, DEBRA, Rt. 1, Box 412, St. Albans, WV 25177.

COCKE, J. M., Department of Geology, Central Missouri State University, Warrensburg, MO 64093.

DiMICHELE, WILLIAM A., Department of Paleobiology, W-310, National Museum of Natural History, Smithsonian Institution, Washington, DC 20560.

EBY, DAVID E., 1324 East Easter Circle, Littleton, CO 80122.

ELDER, WILLIAM P., Department of Geological Sciences, Campus Box 250, University of Colorado, Boulder, CO 80309.

EYLANDS, KURT E., 1965 25th Ave. So., Grand Forks, ND 52801.

FOOTE, MICHAEL J., Department of Geophysical Sciences, University of Chicago, 5734 S. Ellis Ave., Chicago, IL 60637.

FORSEY, G. F., Nene College, Moulton Park, Northampton, NN2 61B, England.

FROST, HOWARD, P.O. Box 711028, Los Angeles, CA 90071.

FRYE-HUAYHUACA, CYNTHIA, 28 Burgett Drive, Homer, NY 13077.

GALLAGHER, WILLIAM B., New Jersey State Museum, 205 West State Street, Trenton, NJ 08625.

GILMORE, JOHN B., 10405 Malaquena N.E., Albuquerque, NM 87111.

GOODMAN, STEPHEN D., 3740 Cloudland Drive, N.W., Atlanta, GA 30327.

HAASE, FRANCIS E., 2197 Cruger Ave., Bronx, NY 10462.

HOLLINGSWORTH, J. STEWART, 559 McMullin Drive, Grand Junction, CO 81504.

JANIS, CHRISTINE MARIE, Division of Biology and Medicine, Brown University, Box 6, Providence, RI 02912.
JOHNSON, D. CHAD, North 3303 Sheridan Ct., Spokane, WA 99205.

JOHNSON, KENNETH G., Department of Geology, University of Iowa, Iowa City, IA 52240.

KHERADYAR, TARA, Department of Paleontology, Earth Science Building, University of California, Berkeley, CA 94720.

LAGARRY, HANNAN E., 212 West 7th St., Apt. 5, Hays, KS 67601.

LAW, ROBERT H., Department of Geology, University of Pennsylvania, Philadelphia, PA 19104.

LEBIADOWSKI, GEORGE, Box 2014, Rocky Mountain House, Alberta T0M-1 T0, Canada.

LEE, YEON GYU, Institute of Geoscience, The University of Tsukuba, Ibaraki, 305, Japan.

MAJIMA, NOBUA, 3-1-51 Chu-Oh, Kuki City, Saitama Pre., Japan.

MCGEE, ELIZABETH M., Department of Paleontology, 295 Earth Sciences Building, University of California, Berkeley, CA 94720.

MCLEOD, JOHN D., 1137 North 26th St., Billings, MT 59101.

METZ, ROBERT, Department of Geology \& Meteorology, Kean College of New Jersey, Union, NJ 07083.

MORALES, MICHAEL A., Museum of Northern Arizona, Rt. 4, Box 720, Flagstaff, AZ 86001.

NAKAYA, HIDEO, Department of Earth Sciences, Kagawa University, Takamatsu, 760, Japan.

OAKES, WAYNE, Box 40361, Albuquerque, NM 87196.

PETZOLD, DANIEL D., 2619 E. Fifth St., Bloomington, IN 47401.

PILETTE, RON, Department of Biology, Brooklyn College, Brooklyn, NY 11210.

POHLER, SUSANNE, Department of Earth Sciences, Memorial University of Newfoundland, St. John's, Newfoundland A1B 3X5, Canada.

RAMOS, RICHARD A., JR., Walsh Hall, Rm. 120, Boston College, Chestnut Hill, MA 02167.

RIDDLE, STEVEN, Department of Geology \& Mineralogy, Ohio State University, 125 South Oval Mall, Columbus, OH 43210.

RITTERBUSH, LINDA ANITA, Department of Geology, California Lutheran University, Thousand Oaks, CA 91360.

ROE, LOIS J., Mt. Holyoke College, South Hadley, MA 01075.

SCHWIMMER, BARBARA A., 3840 Neville Dr., Brimfield, OH 44240.

SHANE, BRUCE S., P.O. Box 17472, Irvine, CA 92713.

SHERWOOD, KATHERINE MARIE, 1517 Reynolds, Laramie, WY 82070. 
SIMMONS, NANCY B., Department of Paleontology, University of California, Berkeley, CA 94720.

SMITH, WARREN A., 308 Larel Lane, Roswell, GA 30076.

SPARLING, DALE R., Earth Science Program, Southwest State University, Marshall, MN 56258.

STANNAGE, WILLIAM, 5 Coastguard Station, Robin Hood's Bay, North Yorkshire YO22 4SY, England.

STORRS, GLEN W., Department of Geology, Yale University, P.O. Box 6666, New Haven, CT 06511.
SUTER, SHERMAN, Department of Geophysical Sciences, University of Chicago, 5734 S. Ellis Ave., Chicago, IL 60637.

TASSELL, C. B., Queen Victoria Museum \& Art Gallery, Wellington Street, Launceston, Tasmania 7250, Australia.

WAMBACK, STEVEN J., 9130 Brown Rd., Angola, NY 14006.

WATSON, JAMES B., Department of Geology, University of Tennessee, Knoxville, TN 37996. WEIS, GERALD H., P.O. Box 1031, Flint, MI 48501 .

WILDUNG, JON R., 330 W. Warren, Luverne, MN 56156. 